\title{
Synthesis of some new indole derivatives containing pyrazoles with potential antitumor activity
}

\author{
Abdel-Rahman Farghaly \\ Department of Chemistry, Faculty of Science, Assiut University, 71516 Assiut, Egypt \\ Current Address: Chemistry Department, Faculty of Science, Jazan University, 2097 Jazan, KSA \\ E-mail: elrahman2001@hotmail.com
}

\begin{abstract}
A series of new [1-(4-methoxybenzyl)indol-3-yl](1H-pyrazol-1-yl)methanones, 1-(1-(4methoxybenzyl)- $1 H$-indole-3-carbonyl)-3-subsituted-1H-pyrazol-5(4H)-ones have been developed using the 1-(4-methoxybenzyl)-1H-indole-3-carbohydrazide $\mathbf{1}$ as a key intermediate. The target compounds were tested in-vitro for tumor cell-growth inhibition.
\end{abstract}

Keywords: Indoles, pyrazoles, pyrazolones, antitumor activity

\section{Introduction}

Indole nucleus is frequently found in medicinal chemistry and is considered as "privileged scaffolds". ${ }^{1}$ Therefore, the synthesis and selective functionalization of indoles have been the focus of active research over the years. ${ }^{2-4}$ Indole derivatives constitute an important class of therapeutic agents in medicinal chemistry including anticancer, ${ }^{5}$ antioxidant, ${ }^{6}$ antirheumatoidal and anti-HIV ${ }^{7,8}$ and also play a vital role in the immune system. ${ }^{9,10}$ Many indole derivatives are considered as the most potent scavenger of free radicals. ${ }^{11}$ Artificial receptors for biologically active molecules have attracted attention from the view point of molecular recognition. ${ }^{12}$

In addition, it was reported that various 3-substituted indoles had been used as starting materials for the synthesis of a number of alkaloids, agrochemicals, pharmaceuticals and perfumes. $^{13}$

On the other hand, over the past two decades, pyrazole-containing compounds have received considerable attention owing to their diverse chemotherapeutic potentials including versatile antineoplastic activities. Literature survey revealed that some pyrazoles have been implemented as antileukemic, ${ }^{14-16}$ antitumor ${ }^{17-20}$ and anti-proliferative ${ }^{21,22}$ agents, beside their capability to exert remarkable anticancer effects through inhibiting different types of enzymes that play important roles in cell division. ${ }^{23-25}$ We have recently reported synthesis and antitumor activity of some indole derivatives containing 1,3,4-oxadiazole and 1,2,4-triazole A, and discovered that 
some of the analogs are potent and selective against various cancer cell lines. ${ }^{26}$ Combination of the pyrazole moiety with the indole nucleus may enhance these activities. In view of these previous findings, intriguing cytotoxicity of various indolyl azoles and in continuation of our interest in the functionalization of indoles (A, B, C and $\mathbf{D}){ }^{29,30}$ and in the development of new synthetic methods, ${ }^{26-31}$ we report herein on the synthesis of some new indole derivatives containing pyrazoles with potential antitumor activity.

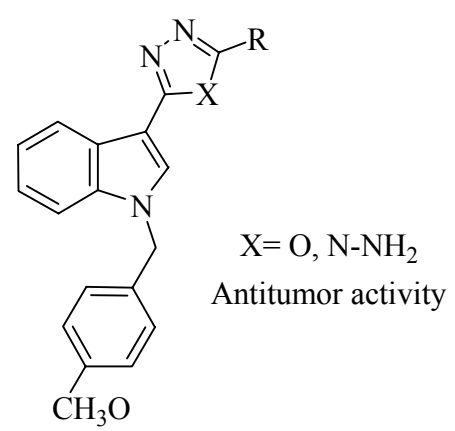

A

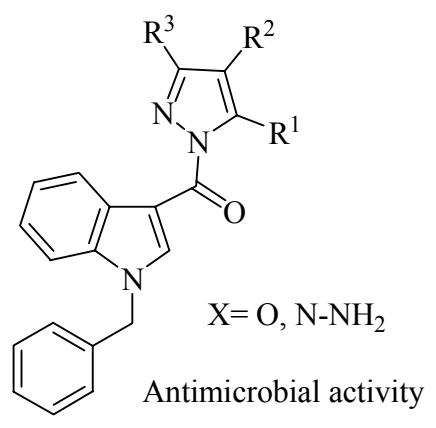

B

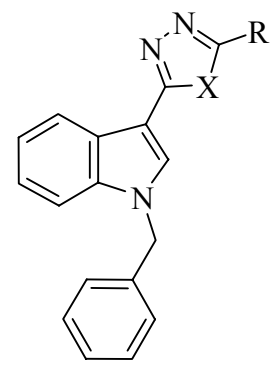

C

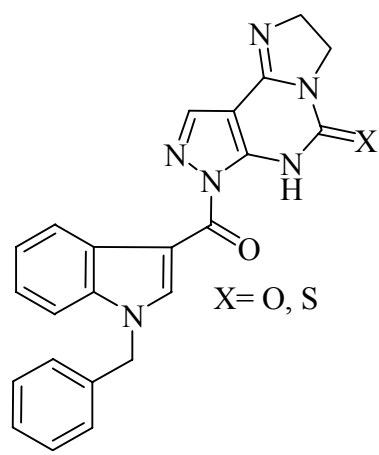

D

Figure 1

\section{Results and Discussion}

The easily accessible 1-(4-methoxybenzyl)-1H-indole-3-carbohydrazide $\mathbf{1}^{26}$ was chosen as starting material of the synthesis of the new pyrazole derivatives as well as pyrazolone derivatives. Thus, treatment of carbohydrazide 1 with ethyl ethoxymethylenecyanoacetate or ethoxymethylenemalononitrile in absolute ethanol resulted in the formation of the corresponding ethyl 5-amino-1-(1-(4-methoxybenzyl)-1H-indole-3-carbonyl)-1H-pyrazole-4-carboxylate 2 and 5-Amino-1-(1-(4-methoxybenzyl)-1H-indole-3-carbonyl)-1H-pyrazole-4-carbonitrile respectively. Analogously, when $\mathbf{1}$ was allowed to react with diethyl ethoxymethylenemalonate in refluxing absolute ethanol, the expected ethyl 5-hydroxy-1-(1-(4-methoxybenzyl)-1H-indole3-carbonyl)-1H-pyrazole-4-carboxylate analog 4 was obtained (Scheme 1).

The structures of compounds 2, 3 and $\mathbf{4}$ were confirmed by their spectral data (IR, ${ }^{1} \mathrm{H}$ NMR, ${ }^{13} \mathrm{C}$ NMR and MS) together with elemental analyses. The IR spectrum of compounds 2, 3 and $\mathbf{4}$ reveals the absence of characteristic absorption bands due to carbohydrazide $\mathrm{NHNH}_{2}$ function and showed new characteristic bands at $\bar{v} 3420,3320,3150$ and $2220 \mathrm{~cm}^{-1}$ due to $\mathrm{NH}_{2}$ and $\mathrm{C} \equiv \mathrm{N}$ respectively. The ${ }^{1} \mathrm{H}$ NMR spectrum of compound 2 expectedly shows characteristic signals near $\delta 4.31,1.37$ assignable to $\mathrm{CH}_{2}$ and $\mathrm{CH}_{3}$ respectively and at $\delta 10.75$ assignable to $\mathrm{OH}$. Further confirmation was achieved by the ${ }^{13} \mathrm{C}$ NMR spectrum which showed signals at $\delta 49.35,55.09$ and 116.67 due to $\mathrm{OCH}_{3}, \mathrm{CH}_{2}$ and $\mathrm{C} \equiv \mathrm{N}$ respectively. Chemical confirmation for the amino ester 2 was achieved by boiling with formamide at $210{ }^{\circ} \mathrm{C}$, the pyrazolo[3,4-d]pyrimidinone 5 
resulted. The formation of the pyrimidinone $\mathbf{5}$ is clearly evidenced by disappearance of the characteristic bands which belongs $\mathrm{NH}_{2}$ and ester groups and showed new characteristic absorption bands at $\bar{v} 3300$ and $1670 \mathrm{~cm}^{-1}$ due to $\mathrm{NH}$ and $\mathrm{C}=\mathrm{O}$ respectively.

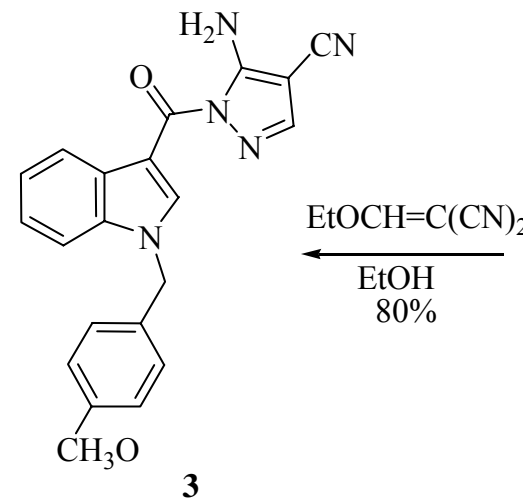

3

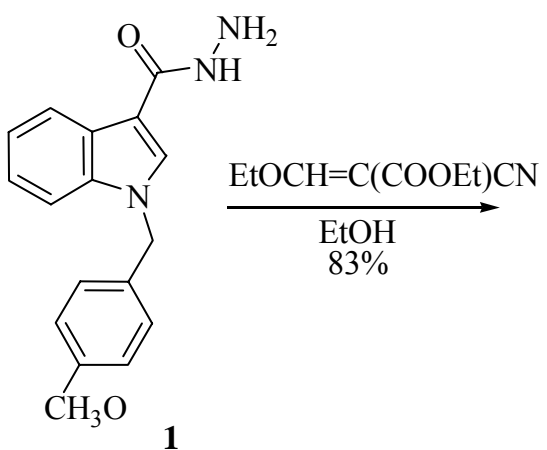

$68 \% \underset{\mathrm{EtOH}}{\mathrm{EtOCH}=\mathrm{C}(\mathrm{COOEt})_{2}}$<smiles>CCOC(=O)c1cnn(C(=O)c2cn(Cc3ccc(C)cc3)c3ccccc23)c1O</smiles>

4<smiles>CCOC(=O)c1cnn(C(=O)c2cn(Cc3ccc(C)cc3)c3ccccc23)c1N</smiles>

$88 \% \downarrow \underset{210{ }^{0} \mathrm{C}}{\mathrm{HCONH}_{2}}$<smiles>Cc1ccc(Cn2cc(C(=O)n3ncc4c(=O)[nH]cnc43)c3ccccc32)cc1</smiles>

\section{Scheme 1}

Treatment of the acid hydrazide 1 with ethyl acetoactetate led to the formation of the corresponding methyl-1H-pyrazol-5(4H)-one 6 in good yield, whereas when it was allowed to react with ethyl benzoylacetate under the same conditions, the open structure was obtained. The later compound could be cyclized via its boiling with high boiling point solvent (propanol) to afford the corresponding $1 H$-pyrazol-5(4H)-one 8 in good yield (Scheme 2). 


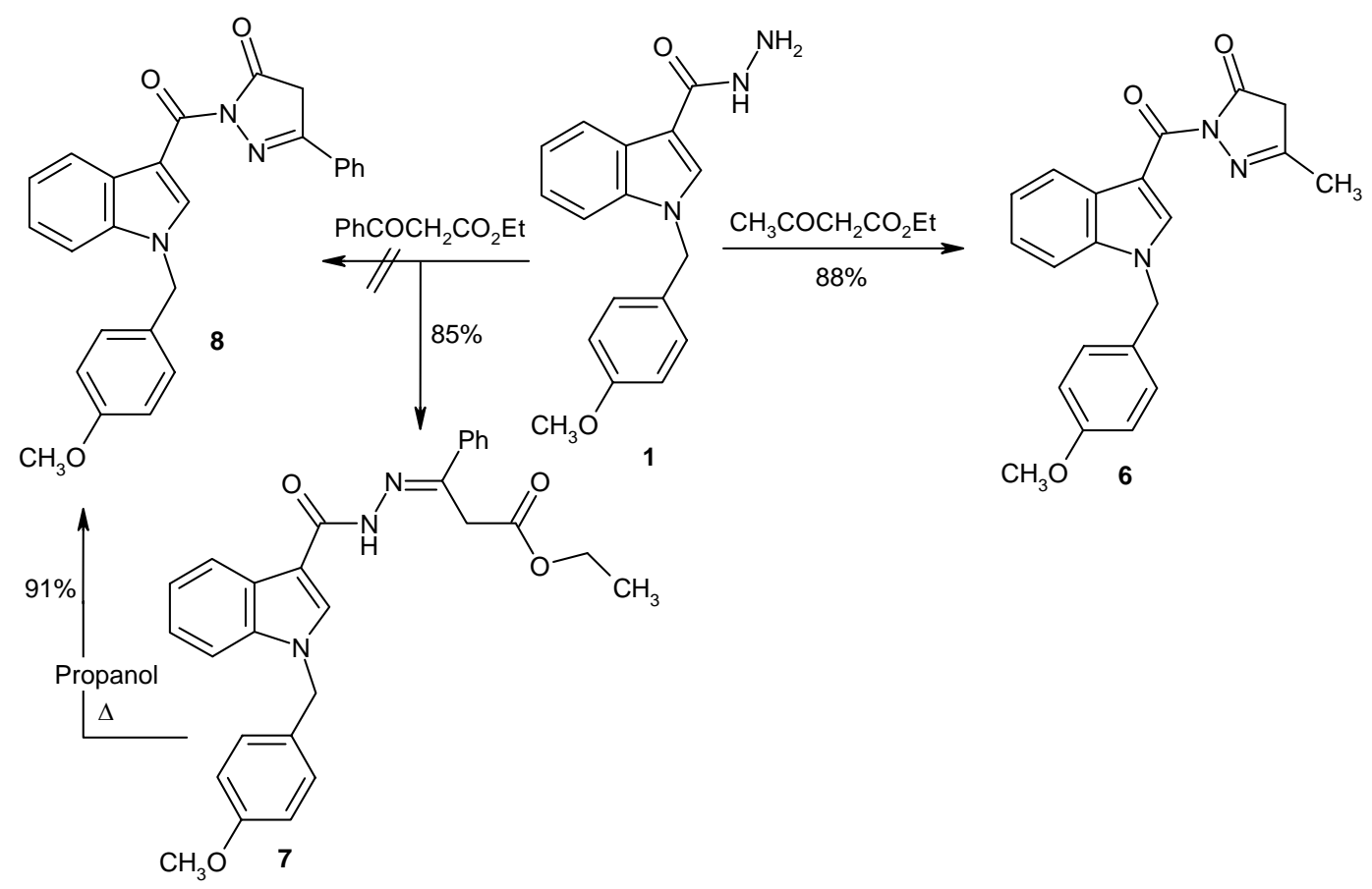

\section{Scheme 2}

The formation of compounds $\mathbf{6}$ and $\mathbf{8}$ was clearly elucidated by characteristic IR absorption bands of the products at $\bar{v} 1660 \mathrm{~cm}^{-1}$ due to $\mathrm{C}=\mathrm{O}$ and disappearance the characteristic absorption bands belonging to $\mathrm{NHNH}_{2}$ group. Further elucidation was done by ${ }^{1} \mathrm{H}$ NMR which showed signals at $\delta 3.52,2.35$ due to $\mathrm{CH}_{2}, \mathrm{CH}_{3}$ and 7.65/7.40 due to phenyl protons respectively. The open structure 7 was elucidated by ${ }^{1} \mathrm{HNMR}$ which showed clearly the signals at $\delta 4.10$ and 1.38 due $\mathrm{CH}_{2}$ and $\mathrm{CH}_{3}$ respectively.

Interestingly, when the acid hydrazide was allowed to react with acetyl acetone in refluxing ethanol for 2 hours afforded directly the cyclized 3,5-dimethyl pyrazole derivative 9 in 64\% yield. Whereas, heating the same acid hydrazide with benzoyl acetone under the same reaction conditions led to the formation the open structure 11. The later compound was cyclized via its boiling in propanol to afford the corresponding (1-(4-Methoxybenzyl)-1H-indol-3-yl)(3-methyl5-phenyl-1H-pyrazol-1-yl)methanone 12 in relatively good yield (Scheme 3).

It is worthy to note that, when the carbohydrazide $\mathbf{1}$ was allowed to react with acetyl acetone in boiling ethanol for prolonged time, an unexpected debenzylation occurred and the $(3,5-$ dimethyl-1H-pyrazol-1-yl)(1H-indol-3-yl)methanone 10 was obtained. The debenzylation and the formation of compound $\mathbf{1 0}$ was confirmed by ${ }^{1} \mathrm{H}$ NMR which revealed the disappearance of the methoxy, phenyl and $\mathrm{CH}_{2}$ signals and showed new signal at $\delta 10.56$ due to $\mathrm{NH}$. Further confirmation for the structure of compound $\mathbf{1 0}$ was obtained by MS which showed the $[\mathrm{M}]^{+}$ion at $m / z 239(2 \%)$. 


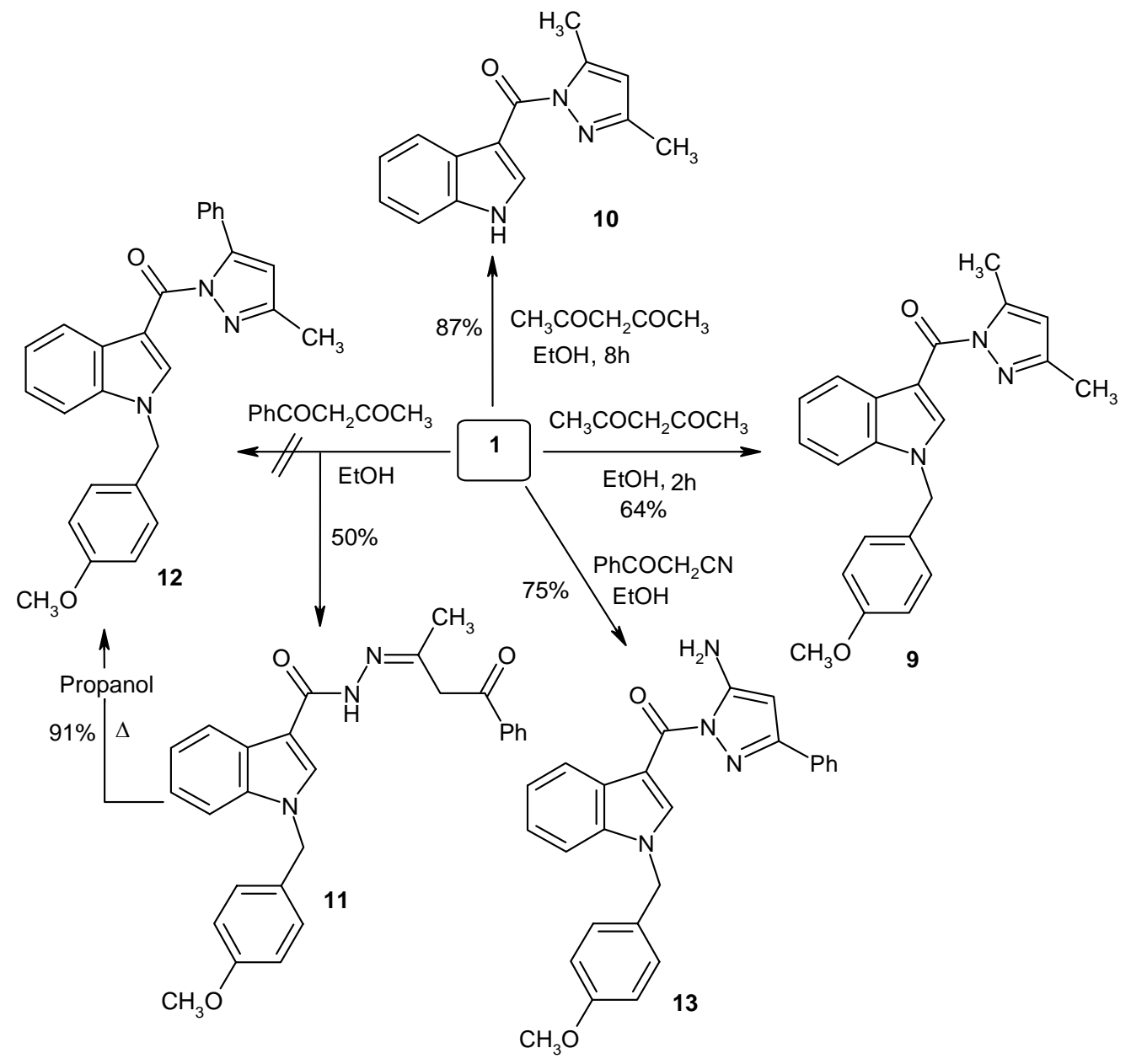

\section{Scheme 3}

Finally, treatment of the acid hydrazide with benzolyl acetonitrile in boiling ethanol rise to the formation of the corresponding 5-amino-3-phenyl-1H-pyrazole 13 in $75 \%$ yield. The IR spectrum of compound 13 showed characteristic absorption bands at $v 3450,3330$ due to $\mathrm{NH}_{2}$.

The biological activity of all synthesized target compounds was tested in vitro for antitumor activity using the Alamar Blue assay ${ }^{32}$ on a panel of five human tumor cell lines at Zentaris, Germany. The cytotoxicity was evaluated on five different cell lines, cervix cancer (KB/HELA), ovarian carcinoma (SK-OV-3), brain cancer (SF-268), nonsmall-cell lung cancer (NCl-H460), and adenocarcinoma colon cancer (RKOP-27). The first screening was carried out at a predefined concentration of $3.16 \mu \mathrm{g} / \mathrm{ml}$. If the compound led to more than $50 \%$ inhibition at this concentration it was evaluated for EC50 mean values (1M) from at least two experiments on those five different cell lines. It turned out that the amino nitrile 3 showed significant cell-growth inhibitory activity $(>50 \%)$ at a fixed concentration of $3.16 \mu \mathrm{g} / \mathrm{mL}$. Subsequent determination of EC50 concentrations from dose-response curves gave valid values for four cell lines (in the case 
of NCI H-460, EC50 was above the highest test concentration). The results are summarized in Table 1.

Table 1. In-vitro antitumor activity (\% cell-growth inhibition at fixed concentration and $\mathrm{EC}_{50}$ values) for synthesized compounds

\begin{tabular}{|c|c|c|c|c|c|c|c|c|c|c|}
\hline \multicolumn{6}{|c|}{ Single point } & \multicolumn{5}{|c|}{ Dose Response } \\
\hline & $\underset{⿱ ㇒}{\stackrel{\Xi}{\Xi}}$ & $\begin{array}{l}\overrightarrow{1} \\
\dot{z} \\
\frac{1}{n}\end{array}$ & $\begin{array}{l}\infty \\
0 \\
\stackrel{1}{1} \\
\stackrel{1}{\sim}\end{array}$ & $\begin{array}{l}0 \\
\stackrel{1}{I} \\
\stackrel{1}{1} \\
\text { Z }\end{array}$ & $\begin{array}{l}\hat{\sim} \\
\hat{\sigma} \\
\frac{v}{2}\end{array}$ & 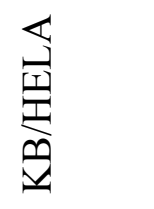 & $\begin{array}{l}\overrightarrow{1} \\
\dot{0} \\
\frac{1}{n}\end{array}$ & $\begin{array}{l}\infty \\
\stackrel{0}{0} \\
\sim \\
\stackrel{1}{\sim}\end{array}$ & 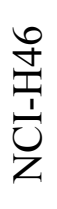 & $\begin{array}{l}\hat{2} \\
\hat{2} \\
\frac{1}{2}\end{array}$ \\
\hline Comp No. & \multicolumn{5}{|c|}{$\% \mathrm{INH}[3.16 \mu \mathrm{g} / \mathrm{ml}]$} & \multicolumn{5}{|c|}{$\mathrm{EC} 50[\mu \mathrm{g} / \mathrm{ml}]$} \\
\hline 2 & -25 & -1 & 5 & 6 & 7 & - & - & - & - & - \\
\hline 3 & 52 & 31 & 34 & 51 & 64 & EC50> & - & - & - & - \\
\hline 7 & -20 & -3 & 5 & -2 & 4 & - & - & - & & \\
\hline 9 & -2 & 0 & 3 & 4 & 9 & & & & & \\
\hline 10 & -2 & 6 & 7 & 18 & 14 & & & & & \\
\hline 11 & 2 & 12 & 14 & 41 & 8 & & & & & \\
\hline 13 & 11 & 7 & 27 & 26 & 25 & & & & & \\
\hline
\end{tabular}

KB/HELA: cervical carcinoma; SK OV-3: ovarial carcinoma; SF-268: CNS cancer; NCIH460: non-small-cell lung cancer; RKOp27: colon adenocarcinoma.

\section{Experimental Section}

General. Melting points were measured on a Kofler melting point apparatus. IR spectra $(\mathrm{KBr}$ pellets) were recorded on a Shimadzu 470 IR-Spectrophotometer. ${ }^{1} \mathrm{H}$ NMR and ${ }^{13} \mathrm{C}$ NMR spectra were obtained using a Varian Gemini-300 (300 MHz for ${ }^{1} \mathrm{H}$, and $75 \mathrm{MHz}$ for ${ }^{13} \mathrm{C}$ ) or a Varian Inova-500 (500 MHz for ${ }^{1} \mathrm{H}$, and $125 \mathrm{MHz}$ for ${ }^{13} \mathrm{C}$ ) spectrometer at the Chemistry Department, Sogang University, Seoul, Korea. Mass spectra were obtained with a Jeol JMS-600 mass spectrometer. Elemental analyses were carried out using a Perkin-Elmer $240 \mathrm{C}$ Micro analyzer and at the Chemistry Department (Microanalytical Laboratory), Assiut University

Ethyl 5-amino-1-(1-(4-methoxybenzyl)-1H-indole-3-carbonyl)-1H-pyrazole-4-carboxylate (2). A mixture of $1(295 \mathrm{mg}, 1 \mathrm{mmol})$ and ethyl ethoxymethylene- cyanoacetate (169 mg, $1 \mathrm{mmol})$ in ethanol $(5 \mathrm{~mL})$ was heated under reflux for $8 \mathrm{~h}$. The progress of the reaction was monitored by HPLC. The solvent was removed under reduced pressure, the residue obtained was collected and recrystallized from ethanol to afford $2(350 \mathrm{mg}, 83 \%)$ as buff crystals, $\mathrm{mp} 135-$ $137{ }^{\circ} \mathrm{C} .{ }^{1} \mathrm{H}$ NMR $\left(500 \mathrm{MHz}, \mathrm{CDCl}_{3}\right) \delta 8.95$ (s, $\left.1 \mathrm{H}, \mathrm{H}-2\right), 8.48(\mathrm{~d}, 1 \mathrm{H}, J=8.70 \mathrm{~Hz}, \mathrm{H}-4), 7.73$ (s, 1H, CH pyrazole), 7.26 (m, 5H, H-5,6,7 and $\left.\mathrm{NH}_{2}\right), 7.12(\mathrm{~d}, J=8.70 \mathrm{~Hz}, 2 \mathrm{H}, \mathrm{ArH}), 6.84(\mathrm{~d}, J=$ 
$8.70 \mathrm{~Hz}, 2 \mathrm{H}, \mathrm{ArH}), 5.34$ (s, 2H, $\mathrm{CH}_{2}$ benzyl), 4.31 (q, $J=7.10 \mathrm{~Hz}, 2 \mathrm{H}, \mathrm{CH}_{2} \mathrm{CH}_{3}$ ), 3.77 (s, 3H, $\left.\mathrm{OCH}_{3}\right), 1.37\left(\mathrm{t}, J=7.10 \mathrm{~Hz}, 3 \mathrm{H}, \mathrm{CH}_{2} \mathrm{CH}_{3}\right) ;{ }^{13} \mathrm{C} \mathrm{NMR}\left(125 \mathrm{MHz}, \mathrm{CDCl}_{3}\right)$ : 165.83, 164.32, $159.53,157.62,154.68,142.41,139.80,136.21,129.08,128.39,127.77,123.62,123.00,122.45$, 114.45, 110.71, 107.15, 103.12, 94.41, 59.85, 55.42, 50.75, 14.69; IR (KBr, cm $\left.{ }^{-1}\right) \bar{v} 3420,3320$, $3150\left(\mathrm{NH}_{2}\right), 2950\left(\mathrm{CH}\right.$ aliph.), 1725, $1690(\mathrm{CO}), 1620(\mathrm{C}=\mathrm{N})$. Anal. Calcd. for $\mathrm{C}_{23} \mathrm{H}_{22} \mathrm{~N}_{4} \mathrm{O}_{4}: \mathrm{C}$, 66.02; H, 5.30; N, 13.39. Found: C, 66.05; H, 5.27; N, 13.47.

5-Amino-1-(1-(4-methoxybenzyl)-1H-indole-3-carbonyl)-1H-pyrazole-4-carbonitrile (3). A mixture of 1 (295 mg, $1 \mathrm{mmol})$ and ethoxymethylene malononitrile (122 mg, $1 \mathrm{mmol})$ in ethanol $(5 \mathrm{~mL})$ was heated under reflux for $8 \mathrm{~h}$. The progress of the reaction was monitored by HPLC. The solvent was removed under reduced pressure and the precipitate obtained was filtered off and recrystallized from ethanol to give $3(200 \mathrm{mg}, 80 \%)$ as white crystals, mp $204-206{ }^{\circ} \mathrm{C} .{ }^{1} \mathrm{H}$ NMR (300 MHz, DMSO-d $)_{6} \delta 8.99$ (s, 1H, H-2), 8.29 (m, 1H, H-4), 8.05 (sb, 2H, NH $), 7.98$ (s, 1H, CH pyrazole), 7.62 (m, 1H, H-7), 7.27 (m, 4H, H-5,6 and 2H ArH), 6.87 (d, J=8.70 Hz, 2H ArH), 5.52 (s, 2H, $\mathrm{CH}_{2}$ benzyl), 3.69 (s, 3H, $\left.\mathrm{OCH}_{3}\right) ;{ }^{13} \mathrm{C}$ NMR (75 MHz, DMSO-d $)$ 164.07, $158.84,155.55,143.56,140.13,135.75,128.81,128.66,128.25,127.34,126.46,123.32,122.67$, 121.43, 116.67, 114.12, 111.64, 105.55, 72.49, 55.09, 49.35; IR $\left(\mathrm{KBr}, \mathrm{cm}^{-1}\right) \vee 3400,3300\left(\mathrm{NH}_{2}\right)$, 2950 (CH aliph.), $2220(\mathrm{C} \equiv \mathrm{N}), 1660(\mathrm{C}=\mathrm{N})$. Anal. Calcd. for $\mathrm{C}_{21} \mathrm{H}_{17} \mathrm{~N}_{5} \mathrm{O}_{2}: \mathrm{C}, 67.91 ; \mathrm{H}, 4.61 ; \mathrm{N}$, 18.86. Found: C, 67.94; H, 4.57; N, 18.78 .

Ethyl 5-hydroxy-1-(1-(4-methoxybenzyl)-1H-indole-3-carbonyl)-1H-pyrazole-4-carboxylate (4). An equimolar mixture of $1(295 \mathrm{mg}, 1 \mathrm{mmol})$, diethyl ethoxymethylene- malonate (225 $\mathrm{mg}$, $1 \mathrm{mmol})$ in absolute ethanol $(10 \mathrm{~mL})$ was refluxed for $10 \mathrm{~h}$. The reaction mixture was concentrated in vacuo, and allowed to attain room temperature. The separated solid product was collected by filtration and recrystallized from ethanol to afford 4 (370 $\mathrm{mg}, 88 \%)$ as buff crystals mp 145-147 ${ }^{\circ} \mathrm{C} .{ }^{1} \mathrm{H}$ NMR $\left(500 \mathrm{MHz}, \mathrm{CDCl}_{3}\right) \delta 10.75$ (s, 1H, OH), 8.97 (s, 1H, H-2), $8.50(\mathrm{~d}, 1 \mathrm{H}$, $J=8.70 \mathrm{~Hz}, \mathrm{H}-4), 7.76$ (s, 1H, CH pyrazole), 7.29 (m, 3H, H-5,6,7), 7.15 (d, $J=8.70 \mathrm{~Hz}, 2 \mathrm{H}$, $\operatorname{ArH}), 6.87(\mathrm{~d}, J=8.70 \mathrm{~Hz}, 2 \mathrm{H}, \mathrm{ArH}), 5.34$ (s, 2H, $\mathrm{CH}_{2}$ benzyl), 4.35 (q, J = $7.10 \mathrm{~Hz}, 2 \mathrm{H}$, $\left.\mathrm{CH}_{2} \mathrm{CH}_{3}\right), 3.75\left(\mathrm{~s}, 3 \mathrm{H}, \mathrm{OCH}_{3}\right), 1.34$ (t, $\left.J=7.10 \mathrm{~Hz}, 3 \mathrm{H}, \mathrm{CH}_{2} \mathrm{CH}_{3}\right) ;{ }^{13} \mathrm{C} \mathrm{NMR}\left(125 \mathrm{MHz}, \mathrm{CDCl}_{3}\right)$ : $166.84,162.35,158.55,157.15,155.65,143.44,138.76,135.29,129.38,128.39,127.67,124.12$, 123.20, 122.15, 115.45, 111.71, 108.15, 102.12, 96.41, 60.85, 55.42, 51.75, 14.69; IR (KBr, cm $\left.{ }^{1}\right)$ v 3460-2720 (OH), 2950 ( $\mathrm{CH}$ aliph.), 1725, $1690(\mathrm{CO}), 1620(\mathrm{C}=\mathrm{N})$. Anal. Calcd. for $\mathrm{C}_{23} \mathrm{H}_{21} \mathrm{~N}_{3} \mathrm{O}_{5}$ : C, 65.86; H, 5.05; N, 10.02. Found: C, 65.78; H, 5.15; N, 9.95

1-(1-(4-Methoxybenzyl)-1H-indole-3-carbonyl)-1H-pyrazolo[3,4- $d$ ]pyrimidin-4(5H)-one (5). A suspension of $2(418 \mathrm{mg}, 1 \mathrm{mmol})$ and formamide $(5 \mathrm{~mL})$ was heated under reflux at $210{ }^{0} \mathrm{C}$ for about $5 \mathrm{~h}$. After cooling, the solvent was removed under reduced pressure and the residue obtained was triturated with water. The solid product formed was filtered off, air dried and recrystallized from ethanol to afford $5(272 \mathrm{mg}, 68 \%)$ as brown crystals, mp $186-188{ }^{\circ} \mathrm{C} .{ }^{1} \mathrm{H}$ NMR (300 MHz, DMSO-d $)_{6} \delta 8.15$ (s, 1H, H-2), 8.12 (d, J = 7.5 Hz, 1H, H-4), 8.03 (sb, 1H, $\mathrm{NH}), 7.98$ (s, 1H, CH pyrazole), 7.95 (s, 1H, CH pyrimidine), 7.52 (d, J = $7.80 \mathrm{~Hz}, 1 \mathrm{H}, \mathrm{H}-7)$, $7.16(\mathrm{~m}, 4 \mathrm{H}$, indole-H5,6, $\mathrm{ArH}), 6.88(\mathrm{~m}, 2 \mathrm{H}, \mathrm{ArH}), 5.36\left(\mathrm{~s}, 2 \mathrm{H}, \mathrm{CH}_{2}\right.$ benzyl), $3.70(\mathrm{~s}, 3 \mathrm{H}$, 
$\left.\mathrm{OCH}_{3}\right)$. IR $\left(\mathrm{KBr}, \mathrm{cm}^{-1}\right) \vee 3300(\mathrm{NH}), 1670(\mathrm{CO})$. Anal. Calcd. for $\mathrm{C}_{22} \mathrm{H}_{17} \mathrm{~N}_{5} \mathrm{O}_{3}$ : C, 66.16; H, 4.29; N, 17.53. Found: C, 66.07; H, 4.23; N, 17.44.

1-(1-(4-Methoxybenzyl)-1H-indole-3-carbonyl)-3-methyl-1H-pyrazol-5(4H)-one (6). A mixture of 1 (295 mg, $1 \mathrm{mmol})$ and ethyl acetoacetate $(5 \mathrm{~mL})$ was heated under reflux for about $8 \mathrm{~h}$. The progress of the reaction was monitored by HPLC. The excess of the solvent was removed under reduced pressure and the residue obtained was collected and recrystallized from ethanol to give 6 (320 mg, 88\%) as buff crystals, mp 145-147 ${ }^{\circ} \mathrm{C} .{ }^{1} \mathrm{H}$ NMR $(300 \mathrm{MHz}$, DMSO$\left.d_{6}\right) \delta 8.28(\mathrm{~s}, 1 \mathrm{H}, \mathrm{H}-2), 7.58\left(\mathrm{~d}, J_{4-5}=8.10 \mathrm{~Hz}, 1 \mathrm{H}, \mathrm{H}-4\right), 7.37(\mathrm{~m}, 4 \mathrm{H}, \mathrm{H}-5,6$ and $2 \mathrm{H} \mathrm{ArH}), 6.97$ $(\mathrm{d}, J=8.70 \mathrm{~Hz}, 2 \mathrm{H} \mathrm{ArH}), 5.61\left(\mathrm{~s}, 2 \mathrm{H}, \mathrm{CH}_{2}\right.$ benzyl), $3.70\left(\mathrm{~s}, 3 \mathrm{H}, \mathrm{OCH}_{3}\right), 3.52\left(\mathrm{~s}, 2 \mathrm{H}, \mathrm{CH}_{2}\right.$ pyrazolone), $2.35\left(\mathrm{~s}, 3 \mathrm{H}, \mathrm{CH}_{3}\right)$; IR $\left(\mathrm{KBr}, \mathrm{cm}^{-1}\right) \vee 1660(\mathrm{C}=\mathrm{O})$. Anal. Calcd. for $\mathrm{C}_{21} \mathrm{H}_{19} \mathrm{~N}_{3} \mathrm{O}_{3}$ : C, 69.79; H, 5.30; N, 11.63. Found: C, 69.68; H, 5.22; N, 11.52.

Ethyl 3-(2-(1-(4-methoxybenzyl)-1H-indole-3-carbonyl)hydrazono)-3-phenyl- propanoate (7). A mixture of $\mathbf{1}(295 \mathrm{mg}, 1 \mathrm{mmol})$ and ethyl benzoylacetate $(5 \mathrm{~mL})$ was heated under reflux for about $8 \mathrm{~h}$. The progress of the reaction was monitored by HPLC. The excess of the solvent was removed under reduced pressure and the residue obtained was collected and recrystallized from ethanol to give 7 (360 mg, 85\%) as white crystals, mp 156-58 ${ }^{\circ} \mathrm{C}$. ${ }^{1} \mathrm{H}$ NMR (500 MHz, $\mathrm{DMSO}_{-} \mathrm{d}_{6} \delta 10.59(\mathrm{~s}, 1 \mathrm{H}, \mathrm{NH}) ; 8.35(\mathrm{~s}, 1 \mathrm{H}, \mathrm{H}-2), 8.23\left(\mathrm{~d}, J_{4-5}=7.80 \mathrm{~Hz}, 1 \mathrm{H}, \mathrm{H}-4\right), 7.95\left(\mathrm{~d}, J_{7-8}\right.$ $=7.80 \mathrm{~Hz}, \mathrm{H}-7) 7.62(\mathrm{~m}, 3 \mathrm{H}, \mathrm{ArH}), 7.41(\mathrm{~m}, 2 \mathrm{H}, \mathrm{ArH}), 7.27$ (d, J=8.10 Hz, 2H ArH), $7.21(\mathrm{~m}$, 2H, H-5,6 indole), 6.89 (d, J=8.10 Hz, 2H ArH), 5.43 (s, 2H, $\mathrm{CH}_{2}$ benzyl), $4.13\left(\mathrm{~s}, 2 \mathrm{H}, \mathrm{CH}_{2}\right)$, 4.10 (q, J= $\left.7.10 \mathrm{~Hz}, 2 \mathrm{H}, \underline{\mathrm{CH}}_{2} \mathrm{CH}_{3}\right), 3.70\left(\mathrm{~s}, 3 \mathrm{H}, \mathrm{OCH}_{3}\right) ; 1.38$ (t, J= $\left.7.10 \mathrm{~Hz}, 3 \mathrm{H}, \mathrm{CH}_{2} \mathrm{C}_{3}\right)$; IR $\left(\mathrm{KBr}, \mathrm{cm}^{-1}\right): v 3200(\mathrm{NH}), 1730(\mathrm{CO}), 1640(\mathrm{C}=\mathrm{N})$; Anal. Calcd. for $\mathrm{C}_{28} \mathrm{H}_{27} \mathrm{~N}_{3} \mathrm{O}_{4}$ : C, 71.62; $\mathrm{H}$, 5,$80 ; \mathrm{N}, 8.95$. Found: C, 71.53; H, 5.74; N, 8.86.

1-(1-(4-Methoxybenzyl)-1H-indole-3-carbonyl)-3-phenyl-1H-pyrazol-5(4H)-one (8). A mixture of 6 (469 mg, $1 \mathrm{mmol})$ in propanol $(10 \mathrm{~mL})$ and few drops of acetic acid was boiled under reflux for about $4 \mathrm{~h}$. The reaction was followed up by HPLC. After cooling, the product formed was collected by filtration and recrystallized from ethanol to give $8(420 \mathrm{mg}, 91 \%)$ as buff crystals, mp. 306-308 ${ }^{\circ} \mathrm{C} .{ }^{1} \mathrm{H}$ NMR $\left(300 \mathrm{MHz}, \mathrm{DMSO}-d_{6}\right) \delta 8.67\left(\mathrm{~d}, J_{4-5}=7.80 \mathrm{~Hz}, 1 \mathrm{H}, \mathrm{H}-\right.$ 4), 8.40 (s, 1H, H-2), 7.90 (d, $\left.J_{7-8}=7.80 \mathrm{~Hz}, \mathrm{H}-7\right) 7.65$ (m, 3H, ArH), 7.40 (m, 2H, ArH), 7.28 (d, $J=8.10 \mathrm{~Hz}, 2 \mathrm{H} \mathrm{ArH}), 7.21(\mathrm{~m}, 2 \mathrm{H}, \mathrm{H}-5,6$ indole), 6.89 (d, $J=8.10 \mathrm{~Hz}, 2 \mathrm{H} \mathrm{ArH}), 5.53$ (s, $2 \mathrm{H}, \mathrm{CH}_{2}$ benzyl), 3.80 (s, 3H, $\left.\mathrm{OCH}_{3}\right), 3.54$ (s, 2H, $\mathrm{CH}_{2}$ pyrazolone); IR $\left(\mathrm{KBr}, \mathrm{cm}^{-1}\right): v 1680$ (CO), $1640(\mathrm{C}=\mathrm{N})$; Anal. Calcd. for $\mathrm{C}_{26} \mathrm{H}_{21} \mathrm{~N}_{3} \mathrm{O}_{3}$ : C, 73.74; H, 5.00; N, 9.92; O, 11.33. Found: C, 73.65; H, 4.90; N, 9.86.

(3,5-Dimethyl-1H-pyrazol-1-yl)(1-(4-methoxybenzyl)-1H-indol-3-yl)methanone (9). A mixture of 1 (295 mg, $1 \mathrm{mmol}$ ) and acetylacetone $(5 \mathrm{~mL})$ was heated under reflux for about $2 \mathrm{~h}$. The progress of the reaction was monitored by HPLC. The excess of the solvent was removed under reduced pressure and the residue obtained was collected and recrystallized from ethanol to give 9 (230 mg, 64\%) as white crystals, mp 252-254 ${ }^{\circ} \mathrm{C} .{ }^{1} \mathrm{H}$ NMR (500 MHz, DMSO- $\left.d_{6}\right) \delta 8.74$ (s, 1H, H-2), 8.56 (d, J4-5 = 8.7 Hz, 1H, H-4), 7.3 (m, 3H, H-5,6,7 indole), 7.14 (d, J = 8.4 Hz, $2 \mathrm{H}, \mathrm{ArH}), 6.87$ (d, $J=8.4 \mathrm{~Hz}, 2 \mathrm{H}, \mathrm{ArH}), 6.05$ (s, 1H, CH pyrazole), 5.34 (s, 2H, $\mathrm{CH}_{2}$ benzyl), 
$3.78\left(\mathrm{~s}, 3 \mathrm{H}, \mathrm{OCH}_{3}\right) ; 2.33,2.25\left(2 \mathrm{~s}, 2 \mathrm{H}, 2 \mathrm{CH}_{3}\right)$. IR (KBr, cm $\left.{ }^{-1}\right): v 2920$ (aliph. $\left.\mathrm{CH}\right) 1670(\mathrm{CO})$; Anal. Calcd. for $\mathrm{C}_{22} \mathrm{H}_{21} \mathrm{~N}_{3} \mathrm{O}_{2}$ : C, 73.52; H, 5.89; N, 11.69. Found: C, 73.47; H, 5.72; N, 11.55 .

(3,5-Dimethyl-1H-pyrazol-1-yl)(1-(1H-indol-3-yl)methanone (10). It was obtained using the same procedure mentioned above but the time of reflux was $8 \mathrm{~h}$ and the crude product formed was recrystallized from ethanol to give $10(210 \mathrm{mg}, 87 \%)$ as white crystals, $\mathrm{mp} 264-266{ }^{\circ} \mathrm{C} .{ }^{1} \mathrm{H}$ NMR (500 MHz, DMSO-d $) \delta 10.56(\mathrm{sb}, 1 \mathrm{H}, \mathrm{NH}), 9.15(\mathrm{~s}, 1 \mathrm{H}, \mathrm{H}-2), 8.25\left(\mathrm{~d}, J_{4-5}=8.1 \mathrm{~Hz}, 1 \mathrm{H}\right.$, $\mathrm{H}-4), 8.01\left(\mathrm{~d}, J_{7-8}=8.1 \mathrm{~Hz}, \mathrm{H}-7\right), 7.34(\mathrm{~m}, 3 \mathrm{H}, \mathrm{H}-5,6), 7.07$ (s, 1H, NH), 6.21 (s, 1H, CH pyrazole), 2.55, $2.26\left(2 \mathrm{~s}, 2 \mathrm{H}, 2 \mathrm{CH}_{3}\right)$. IR ( $\left.\mathrm{KBr}, \mathrm{cm}^{-1}\right) \vee 2920$ (aliph. $\left.\mathrm{CH}\right), 1670$ (CO); Anal. Calcd. for $\mathrm{C}_{14} \mathrm{H}_{13} \mathrm{~N}_{3} \mathrm{O}$ : C, 70.28; H, 5.48; N, 17.56. Found: C, 70.19; H, 5.42; N, 17.48; MS m/z: 239 $\left(\mathrm{M}^{+}, 2 \%\right), 240(15), 241(2)$.

1-(4-Methoxybenzyl)- $N^{\prime}$-(4-oxo-4-phenylbutan-2-ylidene)-1H-indole-3-carbohydrazide (11). A mixture of 1 (295 mg, $1 \mathrm{mmol})$ and benzoyl acetone (162 mg, $1 \mathrm{mmol})$ in ethanol (10 mL) was heated under reflux for about $2 \mathrm{~h}$. The reaction was controlled by HPLC. After cooling, the precipitate formed was filtered off and recrystallized from ethanol/ethyl acetate (1:2) to afford 11 (220 mg, 50\%) as pale yellow crystals, mp 262-264 ${ }^{\circ} \mathrm{C} .{ }^{1} \mathrm{H} \mathrm{NMR}\left(500 \mathrm{MHz}, \mathrm{CDCl}_{3}\right) \delta 8.42(\mathrm{~s}$, $1 \mathrm{H}, \mathrm{H}-2), 8.35$ (d, J4-5 $=8.1 \mathrm{~Hz}, 1 \mathrm{H}, \mathrm{H}-4), 7.48$ (d, $\left.J_{7-8}=8.1 \mathrm{~Hz}, \mathrm{H}-7\right), 7.32$ (m, 3H, ArH), 7.26 (m, 2H, ArH), 7.17 (d, J=8.1 Hz, 2H ArH), 7.10 (m, 2H, H-5,6), 6.86 (d, J=8.1 Hz, 2H ArH), 5.64 (s, 1H, NH exchangeable with $\left.\mathrm{D}_{2} \mathrm{O}\right), 5.32\left(\mathrm{~s}, 2 \mathrm{H}, \mathrm{CH}_{2}\right.$ benzyl), $3.78\left(\mathrm{~s}, 3 \mathrm{H}, \mathrm{OCH}_{3}\right) ; 2.94$ $\left(2 \mathrm{~d}, J=18.6 \mathrm{~Hz}, 2 \mathrm{H}, \mathrm{CH}_{2}\right), 2.09\left(\mathrm{~s}, 3 \mathrm{H}, \mathrm{CH}_{3}\right) ;{ }^{13} \mathrm{C} \mathrm{NMR}\left(\mathrm{CDCl}_{3}\right)$ : 197.98, 164.70, 159.87, $159.85,153.99,153,96,145.13,145.10,136.68,136.53,129.23,129.14,128.88,128.81,124.65$, $123.31,123.19,122.31,119.52,114.82,110.52,108.87,95.22,55.86,53.95,36.83,14.69$; IR $\left(\mathrm{KBr}, \mathrm{cm}^{-1}\right.$ ): $v 3300(\mathrm{NH}), 2920$ (aliph. $\mathrm{CH}$ ), 1700 and $1680(\mathrm{CO})$; Anal. Calcd. for $\mathrm{C}_{27} \mathrm{H}_{25} \mathrm{~N}_{3} \mathrm{O}_{3}$ : C, 73.78; H, 5.73; N, 9.56. Found: C, 73.67; H, 5.62; N, 9.48.

(1-(4-Methoxybenzyl)-1H-indol-3-yl)(3-methyl-5-phenyl-1H-pyrazol-1-yl)methanone (12). A suspension of 11 (439 mg, $1 \mathrm{mmol})$ in propanol $(10 \mathrm{~mL})$ and few drops of acetic acid was boiled under reflux for about $4 \mathrm{~h}$. The reaction was followed up by HPLC. After cooling, the product formed was collected by filtration and recrystallized from ethanol to give 11 (400 mg, 91\%) as buff crystals, mp. $278-280{ }^{\circ} \mathrm{C} .{ }^{1} \mathrm{H}$ NMR $\left(500 \mathrm{MHz}, \mathrm{CDCl}_{3}\right) \delta 8.45$ (s, 1H, H-2), $8.33(\mathrm{~d}$, $\left.J_{4-5}=8.1 \mathrm{~Hz}, 1 \mathrm{H}, \mathrm{H}-4\right), 7.50\left(\mathrm{~d}, J_{7-8}=8.1 \mathrm{~Hz}, \mathrm{H}-7\right), 7.36(\mathrm{~m}, 3 \mathrm{H}, \mathrm{ArH}), 7.28(\mathrm{~m}, 2 \mathrm{H}, \mathrm{ArH}), 7.20$ $(\mathrm{d}, J=8.1 \mathrm{~Hz}, 2 \mathrm{H} \mathrm{ArH}), 7.14(\mathrm{~m}, 2 \mathrm{H}, \mathrm{H}-5,6), 6.88(\mathrm{~d}, J=8.1 \mathrm{~Hz}, 2 \mathrm{H} \mathrm{ArH}), 5.52\left(\mathrm{~s}, 2 \mathrm{H}, \mathrm{CH}_{2}\right.$ benzyl), $3.84\left(\mathrm{~s}, 3 \mathrm{H}, \mathrm{OCH}_{3}\right) ; 2.35\left(\mathrm{~s}, 3 \mathrm{H}, \mathrm{CH}_{3}\right) ;{ }^{13} \mathrm{C} \mathrm{NMR}\left(\mathrm{CDCl}_{3}\right): 165.70,158.85,157.80$, $152.94,151,91,144.13,143.10,138.65,137.52$, 130.87, 130.18, 129.10, 128.83, 128.17, 127.97, $126.49,124.65,123.26,122.31,119.52,114.82,110.52,108.87,105.22,55.36,52.45,13.65$; IR $\left(\mathrm{KBr}, \mathrm{cm}^{-1}\right.$ ): $v 2920$ (aliph. $\mathrm{CH}$ ), $1680(\mathrm{CO})$; Anal. Calcd. for $\mathrm{C}_{27} \mathrm{H}_{23} \mathrm{~N}_{3} \mathrm{O}_{2}: \mathrm{C}, 76.94 ; \mathrm{H}, 5.50 ; \mathrm{N}$, 9.97. Found: C, 76.87; H, 5.58; N, 9.88.

(5-Amino-3-phenyl-1H-pyrazol-1-yl)(1-(4-methoxybenzyl)-1H-indol-3-yl)methanone (13). A mixture of 1 (295 mg, $1 \mathrm{mmol}$ ) and benzoyl acetonitrile $(145 \mathrm{mg}, 1 \mathrm{mmol})$ in absolute ethanol $(10 \mathrm{~mL})$ was heated under reflux for about $6 \mathrm{~h}$. The reaction was controlled by HPLC. After cooling, the precipitate formed was filtered off and recrystallized from ethanol to afford 13 (318 mg, 75\%) as pale yellow crystals, mp 221-223 ${ }^{\circ} \mathrm{C} .{ }^{1} \mathrm{H}$ NMR (500 MHz, DMSO-d $) \delta 9.03$ 
(s, 1H, H-2), 8.36 (d, $\left.J_{4-5}=7.2 \mathrm{~Hz}, 1 \mathrm{H}, \mathrm{H}-4\right), 7.92$ (d, $\left.J_{7-8}=7.2 \mathrm{~Hz}, \mathrm{H}-7\right), 7.76$ (d, J = 8.7 Hz, 2H $\operatorname{ArH}), 7.38$ (m, 7H, indole and $\mathrm{ArH}), 6.98$ (d, J=8.7 Hz, 2H ArH), 5.87 (s, 1H, pyrazole), 5.85 (s, 2H, $\left.\mathrm{NH}_{2}\right), 5.52$ (s, 2H, $\mathrm{CH}_{2}$ benzyl), $3.73\left(\mathrm{~s}, 3 \mathrm{H}, \mathrm{OCH}_{3}\right)$; IR $\left(\mathrm{KBr}, \mathrm{cm}^{-1}\right): v 3450,3330\left(\mathrm{NH}_{2}\right)$, 1670 (CO); Anal. Calcd. for $\mathrm{C}_{26} \mathrm{H}_{22} \mathrm{~N}_{4} \mathrm{O}_{2}$ : C, 73.92; H, 5.25; N, 13.26. Found: C, 73.87; H, 5.20; $\mathrm{N}, 13.19$.

\section{Acknowledgements}

We are grateful to Zentaris GmbH (Frankfurt/Main, Germany) for in-vitro testing of the compounds for antitumor activity.

\section{References}

1. (a) Sundberg, R. J. Indoles; Academic Press: San Diego, 1996. (b) Faulkner, D. J. Nat. Prod. Rep. 2001, 18, 1. (c) Ninomiya, I. J. Nat. Prod. 1992, 55, 541. (d) Denhart, D. J.; Deskus, J. A.; Ditta, J. L.; Gao, Q.; King, H. D.; Kozlowski, E. S.; Meng, Z.; LaPaglia, M. A.; Mattson, G. K.; Molski, T. F.; Taber, M. T.; Lodge, N. J.; Mattson, R. J.; Macor, J. E. Bioorg. Med. Chem. Lett. 2009, 4031.

2. (a) Bandini, M.; Melloni, A.; Umani-Ronchi, A. Angew. Chem., Int. Ed. 2004, 43, 550. (b) Austin, J. F.; MacMillan, D. W. C. J. Am. Chem. Soc. 2002, 124, 1172. (c) Jensen, K. B.; Thorhange, J.; Hazel, R. G.; Jorgensen, K. A. Angew. Chem., Int. Ed. 2001, 40, 160.

3. (a) Srivastava, N.; Banik, B. K. J. Org. Chem. 2003, 68, 2109. (b) Bartoli, G.; Bartolacci, M.; Bosco, M.; Foglia, G.; Giuliani, A.; Marcantoni, E.; Sambri, L.; Torregiani, E. J. Org. Chem. 2003, 68, 4594.

4. (a) Yoshiaki, N.; Masato, Y.; Youichi, I.; Masnobu, H.; Sakae, U. J. Am. Chem. Soc. 2002, 124, 11846; (b) Wenkert, E.; Angell, E. C.; Ferreira, V. F.; Michelotti, E. L.; Piettre, S. R.; Sheu, J. H.; Swindell, C. S. J. Org. Chem. 1986, 51, 2343.

5. Chen, I.; Safe, S.; Bjeldanes, L. Biochem. Pharmacol. 1996, 51, 1069.

6. Suzen, S.; Buyukbingol, E. Il Farmaco. 2000, 55, 246.

7. Buyukbingol, E.; Suzen, S.; Klopman, G. Il Farmaco. 1994, 49, 443.

8. Suzen, S.; Buyukbingol, E. Il Farmaco. 1998, 53, 525.

9. Lieberman, P. M.; Wolfler, A.; Felsner, P.; Hofer, D.; Schauenstien, K. Int. Arch. Allergy Immunol. 1997, 112, 203.

10. Page, D.; Yang, H.; Brown, W.; Walpole, C.; Fleurent, M.; Fyfe, M.; Gaudreault, F.; Onge, S. S. Bioorg. Med. Chem. Lett. 2007, 22, 6183.

11. Chyan,Y. J.; Poeggler, B.; Omar, R. A.; Chain, D. G.; Frangione, B.; Ghiso, J.; Pappolla, M. A. J. Biol. Chem. 1999, 274, 21937.

12. Bolz, I.; May, C.; Spange, S. Arkivoc 2007, (iii), 60. 
13. Kasahara, A. Jpn. Kokai Tokkyo Koho JP 62 1987, 153, 271.

14. Daidone, G.; Maggio, B.; Raffa, D.; Plescia, S.; Schillaci, D.; Raimondi, M. V. Il Farmaco. 2004, 59, 413.

15. Chou, L.-C.; Huang, L.-J.; Yang, J.-S.; Lee, F.-Y.; Teng, C.-M.; Kuo, S.-C. Bioorg. Med. Chem. 2007, 15, 1732.

16. Manetti, F.; Brullo, C.; Magnani, M.; Mosci, F.; Chelli, B.; Crespan, E.; Schenone, S.; Naldini, A.; Bruno, O.; Trincavelli, M. L.; Maga, G.; Carraro, F.; Martini, C.; Bondavalli, F.; Botta, M. J. Med. Chem. 2008, 51, 1252.

17. Li, J.; Zhao, Y. F.; Zhao, X. L.; Yuan, X. Y.; Gong, P. Arch. Pharm. Chem. Life Sci. 2006, 339, 593.

18. Xia, Y.; Dong, Z.-W.; Zhao, B.-X.; Ge, X.; Meng, N.; Shin, D.-S.; Miao, J.-Y. Bioorg. Med. Chem. 2007, 15, 6893.

19. Xia, Y.; Fan, C.-D.; Zhao, B.-X.; Zhao, J.; Shin, D.-S.; Miao, J.-Y. Eur. J. Med. Chem. 2008, 43, 2347.

20. Farag, A. M.; Mayhoub, A. S.; Barakat, S. E.; Bayomi, A. H. Bioorg. Med. Chem. 2008, 16, 881.

21. Schenone, S.; Bruno, O.; Ranise, A.; Bondavalli, F.; Brullo, C.; Fossa, P.; Mosti, L.; Menozzi, G.; Carraro, F.; Naldini, A.; Bernini, C.; Manettic, F.; Botta, M. Bioorg. Med. Chem. Lett. 2004, 14, 2511.

22. Daidone, G.; Raffa, D.; Maggio, B.; Raimondi, M. V.; Plescia, F.; Schillaci, D. Eur. J. Med. Chem. 2004, 39, 219.

23. Warshakoon, N. C.; Wu, S.; Boyer, A.; Kawamoto, R.; Renock, S.; Xu, K.; Pokross, M.; Evdokimov, A. G.; Zhou, S.; Winter, C.; Walter, R.; Mekel, M. Bioorg. Med. Chem. Lett. 2006, 16, 5687.

24. Huang, S.; Lin, R.; Yu, Y.; Lu, Y.; Connolly, P. J.; Chiu, G.; Li, S.; Emanuel, S. L.; Middleton, S. A. Bioorg. Med. Chem. Lett. 2007, 17, 1243.

25. Zhu, G.-D.; Gong, J.; Gandhi, V. B.; Woods, K.; Luo, Y.; Liu, X.; Guan, R.; Klinghofer, V.; Johnson, E. F.; Stoll, V. S.; Mamo, M.; Li, Q.; Rosenberg, S. H.; Giranda, V. L. Bioorg. Med. Chem. 2007, 15, 2441.

26. Farghaly, A.; Haider, N.; Lee, D. Arkivoc 2010, submitted.

27. Farghaly, A.; De-clercq, E.; El-Kashef, H. Arkivoc 2006, (x), 137.

28. Farghaly, A.; El-Kashef, H. S. Arkivoc 2006, (xi), 76.

29. Farghaly, A.; El-Kashef, H. Monatsh. Chem. 2006, 137, 1195.

30. Farghaly, A. A. H. J. Chin. Chem. Soc. 2004, 51, 147.

31. Baraldi, P. G.; El-Kashef, H.; Farghaly, A.; Vanelle, P.; Fruttarolo, F. Tetrahedron 2004, 60, 5093.

32. Ahmed, S. A.; Gogal, R. M.; Jr.; Walsh, J. E. J. Immunol. Methods 1994,170, 211. 